\title{
DESIGN OF LOOSELY COUPLED MAGNETIC SYSTEMS BASED ON FINITE ELEMENT METHOD FOR INDUCTIVE POWER TRANSFER APPLICATIONS
}

\author{
Rodolfo Castanho Fernandes, Azauri Albano de Oliveira Jr. \\ Escola de Engenharia de São Carlos, USP - Universidade de São Paulo, Depto. de Engenharia Elétrica e de Computação. \\ São Carlos - SP, Brasil \\ e-mail: rodolfocf@ieee.org
}

\begin{abstract}
This paper proposes an iterative approach for the design of loosely coupled inductors for inductive power transfer applications. The procedure is based on the finite element method and is suitable for realistic coil geometry development where non-linear magnetic materials are required. Dedicated algorithms represent self-inductances and mutual inductances in terms of equivalent geometric parameters and vice-versa thus allowing an approach that is not possible in analytical formulations. Also, finite element method is used to analyze the coil system, with two or more coils, under axial, lateral and angular misalignment prior to the development of prototypes. The iterative method can be used to support development of wireless power converters for biomedical implants, electric vehicles recharging systems and chargers for portable devices, with simple and minor modifications. Also, it has specific tools for geometry optimization that can lead to maximized power transfer. Experimental results are presented for coupling coefficient under spatial misalignment.
\end{abstract}

Keywords - Biomedical Implants, Electrical Vehicles, Finite Element Method, Inductive Power Transfer, Portable Devices.

\section{INTRODUCTION}

The terms Wireless Power Transfer (WPT) and Inductive Power Transfer (IPT) are not recent in literature and the first experiments aiming to transfer significant power through air are dated from the last decades of $19^{\text {th }}$ century [1]. This is especially interesting in the historical context that Maxwell's Equations where compiled just a few years before, in 1860's.

By eliminating solid contacts between source and load, more mobility is allowed and this is the main reason for continued research on IPT techniques. Other advantages of Inductive Power Transfer over conventional power transfer are: 1) No electrical sparks between primary and secondary circuits; 2) Primary and secondary can be encapsulated (e.g. epoxy), thus achieving high insulating level; 3) Less maintenance as fundamentally there are no mechanical contacts such as brushes and slip rings; 4) No restrictions for use in explosive or hazardous areas; 5) Possibility to transfer power through air, liquids or solid walls [2].

One possible approach to understand the IPT concept starts by the analysis of the circuit in Figure 1, which represents a conventional non-ideal transformer driven by a

Manuscript received 04/02/2014; revised 14/11/2014; accepted for publication 01/12/2014, by recommendation of the Special Section Editor Mário Lúcio da Silva Martins. sinusoidal current source. In practical IPT converters, the current source is obtained by means of resonant converters [3], which are basically associations of a high-frequency current-sourced or voltage-sourced square wave inverter and a properly selected LC resonant network.

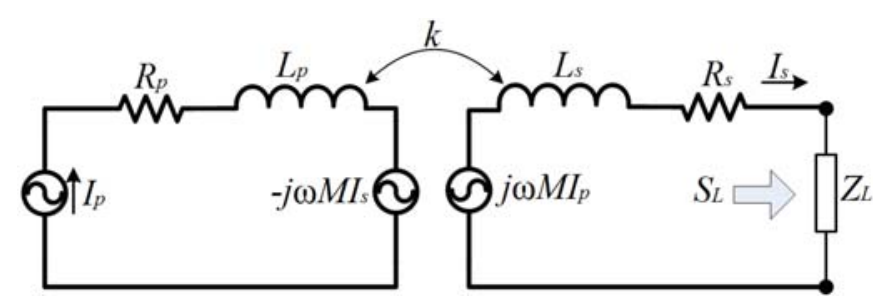

Fig. 1. Basic non-ideal transformer circuit.

Under steady state conditions, current is sinusoidal and it is possible to use phasor notation while frequency is fixed. Thus, considering Figure 1, secondary current $I_{s}$ in phasor form can be written as $I_{s}=\left(j \omega M I_{p}\right) /\left(Z_{s}+Z_{L}\right)$ where $\omega$ is the angular frequency, $M$ is the mutual inductance, $I_{p}$ is the phasor primary current and $Z_{s}$ and $Z_{L}$ are respectively the secondary impedance and the load impedance. Separating $Z_{s}$ and $Z_{L}$ in order to emphasize real and imaginary parts gives $Z_{s}+Z_{L}=\left(R_{s}+R_{L}\right)+j \omega\left(L_{s}+L_{L}\right)$ and, considering that primary current is the reference excitation, (1) is obtained, where $\hat{I}_{p}$ is the peak primary current:

$$
I_{s}=\frac{\omega^{2} M \hat{I}_{p}\left(L_{s}+L_{L}\right)+j \omega M \hat{I}_{p}\left(R_{L}+R_{s}\right)}{\left(R_{L}+R_{s}\right)^{2}+\omega^{2}\left(L_{s}+L_{L}\right)^{2}} .
$$

The apparent power transferred to the load is given by the alternative formulation $S_{L}=Z_{L} I_{S} I_{s}{ }^{*}$. Using $M=k\left(L_{p} L_{s}\right)^{1 / 2}$ in (1), the apparent power is given by (2), where $P_{L}$ and $Q_{L}$ are the active and reactive power delivered to the load:

$$
S_{L}=\frac{\omega^{2} k^{2} L_{p} L_{s} \hat{I}_{p}^{2}\left(R_{L}+j \omega L_{L}\right)}{\left(R_{L}+R_{s}\right)^{2}+\omega^{2}\left(L_{s}+L_{L}\right)^{2}}=P_{L}+j Q_{L} .
$$

One may observe that (2) is derived from the basic circuit model shown in Figure 1 and does not include effect of parasitic capacitances, for example. Thus, equations obtained here are generally suitable for frequencies up to $1 \mathrm{MHz}$.

In a conventional transformer, coupling coefficient $k$ is typically close to unity (usually $k>0.95$ ). By removing the high permeability magnetic core, emitter and receiver circuits become loosely (or weakly) coupled so that $0<k<0.5$ defines a weak coupling range [4]. With such a low coupling coefficient, the power transfer can only be successful if the current circulating in the emitter coil is at high frequency or if high input current amplitude is achieved. Practical IPT 
converters need a balanced combination of both solutions, since semiconductor technologies limit the frequency increase at certain power levels.

Although (2) is an unusual expression for apparent power in a transformer, the dependence on $I_{p}$ and $\omega$ is somehow intuitive and expected. Less evident, however, is the dependence of $S_{L}$ in respect to $k^{2}$. This strongly indicates that, in high performance converters, not only electrical parameters and topologies must be correctly chosen: improved magnetic design is also mandatory since it determines the coupling factor at a given relative spatial position between emitter and receiver or under dynamical variations of $k$ (that are likely to occur in electric vehicles, consumer electronics or biomedical applications due to natural relative movement).

It is a fact, however, that the effects of coupling coefficient over power transfer in a given IPT converter are barely understood in most cases. Thus, it is not surprising that lots of converter solutions are designed to operate with fixed $k$, which is a very unrealistic condition. This happens because a simple spatial characterization method for loosely coupled inductors is lacking and those who attempt to solve this task usually end up in complex and time-demanding analytical approaches, such as Neumann's formula.

Neumann's formula as shown in (3) is an exact approach to determine mutual inductance between two current filaments at any spatial position. In this case, $\mu_{0}$ is the freespace magnetic permeability, $r$ is the distance between coils and $d s$ and $d s$ ' are the incremental sections of the filament wire [5]. For a simple system comprising two coaxial circular filaments of radius $D_{p} / 2$ and $D_{s} / 2$, being $b$ the distance between their centers, $M$ is given as (4). Angles $\varphi$ and $\varphi$ ' result from the chosen filament geometry parametrization.

$$
\begin{aligned}
& M=\frac{\mu_{0}}{4 \pi} \iint \frac{\overrightarrow{d s} \cdot \overrightarrow{d s^{\prime}}}{r} \\
& M=\frac{\mu_{0}}{4 \pi} \int_{0}^{2 \pi 2 \pi} \int_{0}^{\frac{D_{p} D_{s}}{4} \cos \left(\varphi-\varphi^{\prime}\right) d \varphi d \varphi^{\prime}} \frac{\frac{D_{p}^{2}}{4}+\frac{D_{s}{ }^{2}}{4}+b^{2}-\frac{D_{s}}{2} \cos \left(\varphi-\varphi^{\prime}\right)}{\sqrt{\frac{D^{2}}{4}}}
\end{aligned}
$$

Although exact, (3) requires the solution of elliptic integrals, a time consuming task that, for practical engineering design, requires the aid of computational solvers. Other approaches to evaluate coupling coefficient are the tabulated Grover [6] and Nagaoka methods and empirical formulations such as the Wheeler Equation. In common, all these methods are limited to very particular geometries mostly for air-cored inductors and were typically obtained with assumptions such as homogenous magnetic medium, linear magnetic materials and continuous cores. Thus, if applied, these methods result in several restrictions to the design of loosely coupled systems.

To overcome these limitations, the present work proposes a set of computational tools based on the Finite Element Method (FEM) to help and guide the development of loosely coupled inductors system regardless of the emitter or receiver drive circuit given that a sinusoidal excitation is provided.
This package comprises routines developed in MatLab programming language and results in a pre and a postprocessor for Finite Element Method Magnetics (FEMM), which is a well-known free FEM software largely employed in academic researches. Since it is based on a numerical method, the proposed procedure provides approximate solution, but high-complexity geometries, commonly found in realistic IPT applications, can be considered.

Thus, besides of the introduction of a new method for loosely coupled systems development, this work also has as contribution the fact that these computational procedures can be used without previous knowledge on finite element method.

This paper is organized as follows: Section II describes the proposed iterative design procedure with FEM formulation and computational routine details. Section III presents experimental results and non-trivial analysis examples obtained with the proposed approach focused on spatial misalignment conditions. Section IV presents an example of coil optimization while Section V describes another useful tool: the magnetic field mapping algorithm. Finally, Section VI is dedicated to final comments and conclusions.

\section{ITERATIVE DESIGN BASED ON FINITE ELEMENT METHOD}

\section{A. Finite Element Method Formulation}

There are different Finite Element Method formulations and particularities depending on the kind of problem to be solved. In the present work a 2D formulation focused on magnetostatic problems is used and it allows the analysis of planar and axisymmetric geometries. These two representation forms are able to deal with the majority of IPT coil systems. For very specific geometries without radial symmetry line or plane symmetry, it is usually possible to include simplifications and special boundary conditions to perform a basic analysis. The $2 \mathrm{D}$ formulation is also chosen since it often requires much less computational time to achieve a numerical solution when compared to $3 \mathrm{D}$ formulation, thus being suitable for personal computers.

Moreover, FEMM already uses the desired 2D formulation in its solver and allows some integration with $M a t L a b$ via programming language. Instead of developing a FEM processor, the iterative method was developed to interact with FEMM software and use it for geometry discretization (mesh generation), processing and postprocessing. MatLab routines store data, process it, controls the iterative method parameters and literally edits geometries in the finite element pre-processor (add/remove geometric entities such as lines, nodes, arcs and edit they properties as needed). Also, MatLab provides a user-friendly interface for the designer (of whom no previous knowledge on Finite Element Method is required).

For reference and to understand the limitations of the numerical method, mathematical formulation is presented. The magnetic flux density vector, $B$, is given in (5) as a function of the magnetic vector potential, $A$. Substituting (5) in differential form of Faraday's Law of Induction results in 
(6), which can be integrated to yield (7). Finally, considering Ohm's Law in vector notation, (8) is written. $E$ is the electric field intensity vector, $J_{s r c}$ represents current sources, $V$ is a scalar denoting voltage, $\mu(B)$ indicates that non-linear magnetic permeability is taken into account and $\sigma$ the electrical conductivity [7].

$$
\begin{gathered}
\vec{B}=\vec{\nabla} \times \vec{A} \\
\vec{\nabla} \times \vec{E}=-\frac{\partial}{\partial t}(\vec{\nabla} \times \vec{A}) \\
\vec{E}=-\frac{\partial \vec{A}}{\partial t}-\vec{\nabla} V \\
\vec{\nabla} \times\left(\frac{1}{\mu(B)} \vec{\nabla} \times \vec{A}\right)=-\sigma \frac{\partial \vec{A}}{\partial t}+J_{s r c}-\sigma \vec{\nabla} V
\end{gathered}
$$

This last equation is evaluated by FEMM using the variational method and first-order triangle elements to solve $\Omega_{2 \mathrm{D}}$, which is the simulation domain in two dimensions. With minor modifications, (8) is used by the software in time-harmonic problems with one single frequency (which is enough for IPT since resonant converters in fact should provide sinusoidal excitation to the emitter coil at the resonant frequency only).

One might observe that the above formulation ignores displacement currents and as a result, FEMM can only be applied if this effect is negligible. Fortunately, this is the case of IPT converters which employ good electrical conductors and satisfy the condition $\sigma>>\varepsilon_{\text {med }}$ [8], where $\varepsilon_{\text {med }}$ is the electrical permittivity of the medium (typically air). For instance, consider permittivity of vacuum $\varepsilon_{0}=8.85 \times 10^{-12}$ F.m ${ }^{-1}$, relative permittivity of air $\varepsilon_{r}=1, \varepsilon_{\text {med }}=\varepsilon_{0} \varepsilon_{r}$, and for copper $\sigma=5.96 \times 10^{7} \mathrm{~S} . \mathrm{m}^{-1}$. For $\omega=2 \pi \mathrm{f}$, with frequency ranging from $60 \mathrm{~Hz}$ to $1 \mathrm{MHz}$, there is $3.34 \times 10^{-9} \leq \omega \varepsilon_{\text {med }} \leq$ $5.56 \times 10^{-5}$. Thus, $\sigma>>\omega \varepsilon_{\text {med }}$.

\section{B. Possible Problem Approaches}

As said, the introduction of FEM allows simulation of loosely coupled systems with more realistic parameters. This brings new possibilities for converter design, namely the Reverse and Direct Problem approaches, shown in Figure 2 where $\phi_{m}$ accounts for the mutual magnetic flux, $\phi_{d}$ is the leakage flux, $L_{x}$ stands for self-inductance, $i_{x}(t)$ is the sinusoidal excitation or load current, $D_{x}$ is the coil diameter, $E_{x}$ is the coil height, $N_{x}$ is the number of turns, $e$ is the axial separation distance, $d_{c}$ is the lateral separation distance and $\alpha$ is the angular position. Above, $x=p$, if the property refers to emitter coil (primary) and $x=s$ if receiver coil (secondary). This notation will be used from now on for sake of simplicity.

The Reverse Problem is that where the design initiates from the geometrical parameters such as coils diameters and heights, presence of shielding and its materials (and number of layers) and relative spatial position. Outputs of the Reverse Problem approach are the equivalent electrical parameters such as receiver open circuit voltage (for a given emitter sinusoidal excitation), receiver short circuit current, self-inductances $L_{x}$ and mutual inductances $M$ and winding quality factors $Q_{x}$ (which can be obtained with enough accuracy since FEM provides a reliable prediction of conductor length and resulting series resistance $R_{x}$ ). This approach is useful when mechanical restrictions such as maximum converter dimensions apply. Also, it is used when user desires to evaluate the power that can be transferred under certain excitation for a given emitter-receiver relative spatial condition.

An opposite approach, the Direct Problem, is the one in which electrical circuit parameters are known and geometrical representation is desired. To run this mode, excitation currents $i_{x}(t)$ under the desired coupling factor $k$ must be provided by the user along with the self-inductances. These excitations are promptly obtained from any electrical circuit simulation software considering load conditions, power electronics converter topology and control strategy, if any. Thus, the Direct Problem is useful when the basic electrical parameters are known and it can be employed to characterize an existing converter since coil quality factor and emitter-receiver equivalent spatial position are promptly determined.

Currently the iterative routines solve the Direct Problem considering circular coil or spiral coils (pancake). In any of these cases, the number of turns of the windings or the desired coil diameter is entered by the user. In any situation, magnetic shielding can be added and user has full control over the shield material, its thickness and number of layers (due to the complexity of the algorithm, the package currently is able to handle a maximum of two shielding layers of any material, linear or non-linear).

Figure 3 shows a schematic receiver coil with shielding to illustrate that a large number of details are user-defined during iterative procedures. These parameters also include number of wire strands, wire gauge and material properties such as electrical conductivity and magnetic permeability.

It is noteworthy that electrical circuit simulation software can be used to include realistic parameters of semiconductors in those stages that come before the emitter coil or after the receiver coil.
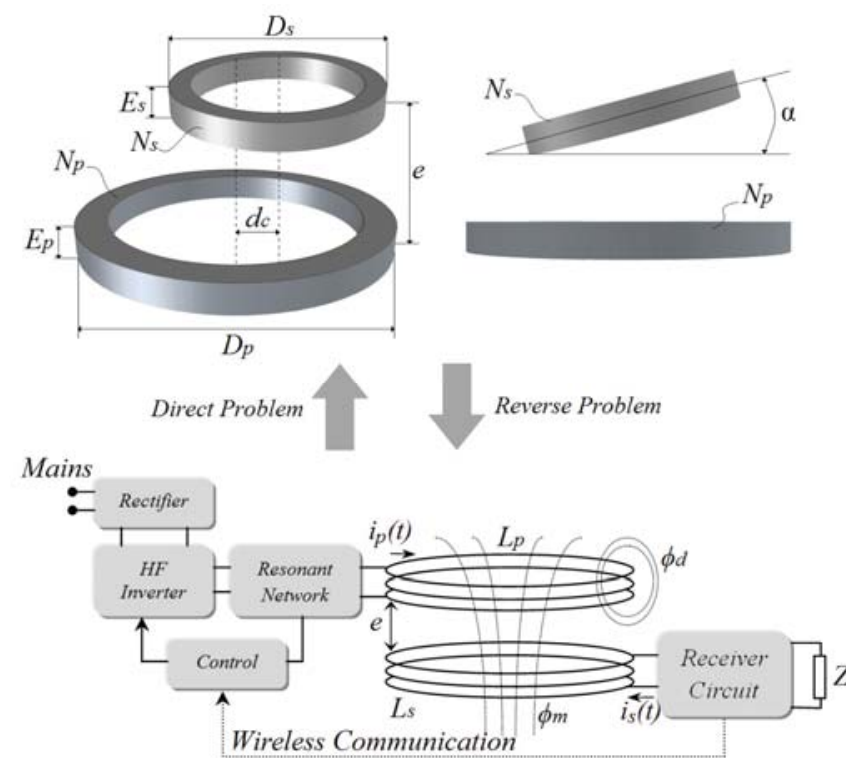

Fig. 2. Direct and Reverse problems. 
One can freely choose the rectifier and inverter topology, semiconductor technology, the kind of resonant network, resonant frequency and the load connected to the secondary side. No matter which power electronics topology is chosen, the iterative method can be applied, if sinusoidal excitation is guaranteed.

\section{Iterative Routines}

Routines are said to be iterative because they rely on computational loops where multiple iterations are performed in order to achieve a desired precision for the target parameter, which can be, for example, $D_{x}$ or $N_{x}$ in the Direct Problem approach or $L_{x}$ in Reverse Problem approach. The procedure is also said to be interactive, since user is able to choose between many options during a routine execution.

Now, assuming that a Direct Problem is requested, four possible routines exist. The user has initially two options: to use spiral coils (which allows the analysis of planar transformers) or circular coils. For any option chosen, other two situations are possible: to solve the problem with fixed coil diameter (this leads to successive modifications of the coil number of turns $N_{x}$ until the target self-inductance $L_{x}$ is obtained) or with fixed number of turns (allowing the coil diameter $D_{x}$ to be modified as necessary). An important note is that these two cases (fixed $D_{x}$ or fixed $N_{x}$ ) are fundamentally different in practical aspects, since the first one imposes a maximum diameter (useful if the converter has critical dimension restrictions) and the second has no mechanical restriction.

Other important note is that since inductance is a function of $N_{x}$, relative magnetic permeability of the medium $\mu_{r}, D_{x}$, $E_{x}$ and winding shape (spiral, toroidal, circular), there is virtually an infinite number of possible inductor geometries that result in the same self and mutual inductances.

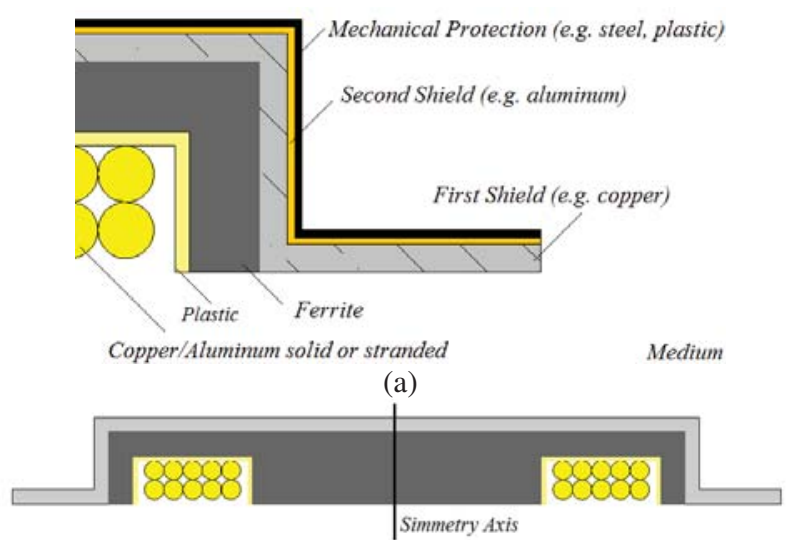

(b)

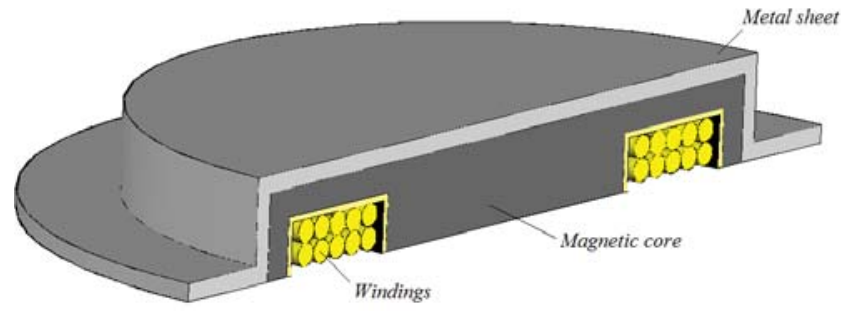

(c)

Fig. 3. Illustration showing possible user-defined parameters in proposed realistic design method (circular coil shown), (a) layers, (b) cross-section and (c) 3D equivalent.

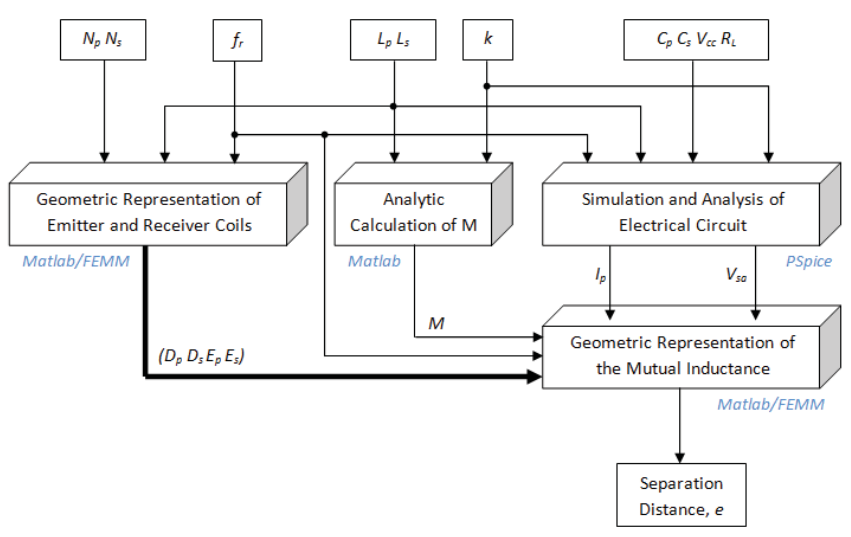

Fig. 4. Matlab/FEMM/PSpice integration block diagram.

That said, at least one restriction must be applied in order to begin the iterative procedure (in fact, this is the biggest problem when analytical approaches are used since too many parameters must be included in the formulation).

The Matlab/FEMM/PSpice integration is represented by Figure 4, where PSpice was chosen as the circuit simulation tool due to its accurate model of semiconductors and commercially available electronic parts. Note that the block designated by "Simulation and Analysis of Electrical Circuit" in Figure 4 may contain any rectifier, inverter or resonant network topology. The important is to guarantee that resulting excitation current is sinusoidal or close to this.

One of the advantages of the formulation shown in (5) is that flux lines are simply plots of the magnetic potential vector. Thus, inductance (self and mutual) is easily obtained as $L_{x}=\phi_{m} / \hat{I}_{x}$, being $\hat{I}_{x}$ the peak excitation current, since $\phi_{m}$ is given according to:

$$
\phi_{m}=\oint_{C} \vec{A} \cdot d l .
$$

This indicates that the line integral is taken over the boundary of the surface, defined by the target coil geometry. The boundary is here denoted by $C$, while $d l$ is the differential straight conductor filament.

In addition, the following variables, included in Figure 4, are defined as:

$C_{x}$ - Compensation capacitances;

$E_{x}-$ Coil height;

$f_{r}$ - Resonant frequency;

$V_{c c}$ - DC bus average voltage;

$R_{L}$ - Load resistance connected to the secondary;

$V_{s a}-$ Secondary open circuit voltage.

All this said, the particularities of each iterative routine are explained as follows.

1) Lumped circular coils, fixed number of turns - This procedure, shown in Algorithm I, seeks for the target inductance $\mathrm{L}_{\mathrm{x}, \text { target }}$ when the user defines the number of turns $N_{x}$. Since $N_{x}$ is fixed and a lumped coils is being designed, the algorithm draws a circle of diameter $E_{x}$ that contains all $N_{x}$ turns (each one with the properties obtained from a winding design routine, not depicted here) with a winding factor $0.7 \leq W_{x} \leq 0.8$. $W_{x}=0.75$ means that $75 \%$ of the 
winding cross section is filled up by copper and $25 \%$ is filled by air. Values between $70 \%$ and $80 \%$ usually guarantee simultaneously a good area usage and practical implementation. This is shown in steps $<4.1>$ to $<4.6>$. Figure 5(a) illustrates the $W_{x}$ adjust loop with a fixed step $\delta_{W}$ (e.g. $\left.\delta_{W}=0.1 \mathrm{~mm}\right)$.

With the winding cross section defined, the only variable that can be used to pursue $L_{x, \text { target }}$ is the coil diameter, $D_{x}$, which is varied based in a diameter step $\Delta D_{x}{ }^{(i-1)}$, defined in the previous iteration $(i-1)$ considering $\varepsilon_{\text {calc }}{ }^{(i)}$, (the error between $L_{x, \text { target }}$ and calculated $L_{x}$ ) and also a pre-defined scalar error gain $\lambda_{L}$, that ensures fast convergence. Do not confuse tolerance $\varepsilon$ with electrical permittivity.

When iteration counter $i$, reaches a maximum iteration number without a final solution for $L_{x, \text { target }}, \lambda_{L}$ is updated in order to force the algorithm to continue. All this define the main loop, as shown in steps $<7.1>$ to $<7.9>$. Figure 5(b) illustrates the main loop, that is finalized when $\varepsilon_{\text {calc }}{ }^{(i)}$ is less that a given precision, $\varepsilon_{\max }$. At this moment, resulting inductor series resistance $R_{x}$ and unloaded quality factor $Q_{x}=$ $\omega L_{x} / R_{x}$ are calculated from the FEM solver.

All inductance calculations are performed using the magnetic materials desired and defined by the user in step $<5.1>$ to $<5.5>$.

When it comes to the time to design the secondary coil, magnetic materials of the primary (if any) are neutralized to avoid their influence over the secondary reluctance path. Thus, one can note that this algorithm seek for the target selfinductance without considering the effect of a nearby structure.

\section{ALGORITHM}

\section{LUMPED CIRCULAR COILS, FIXED N}

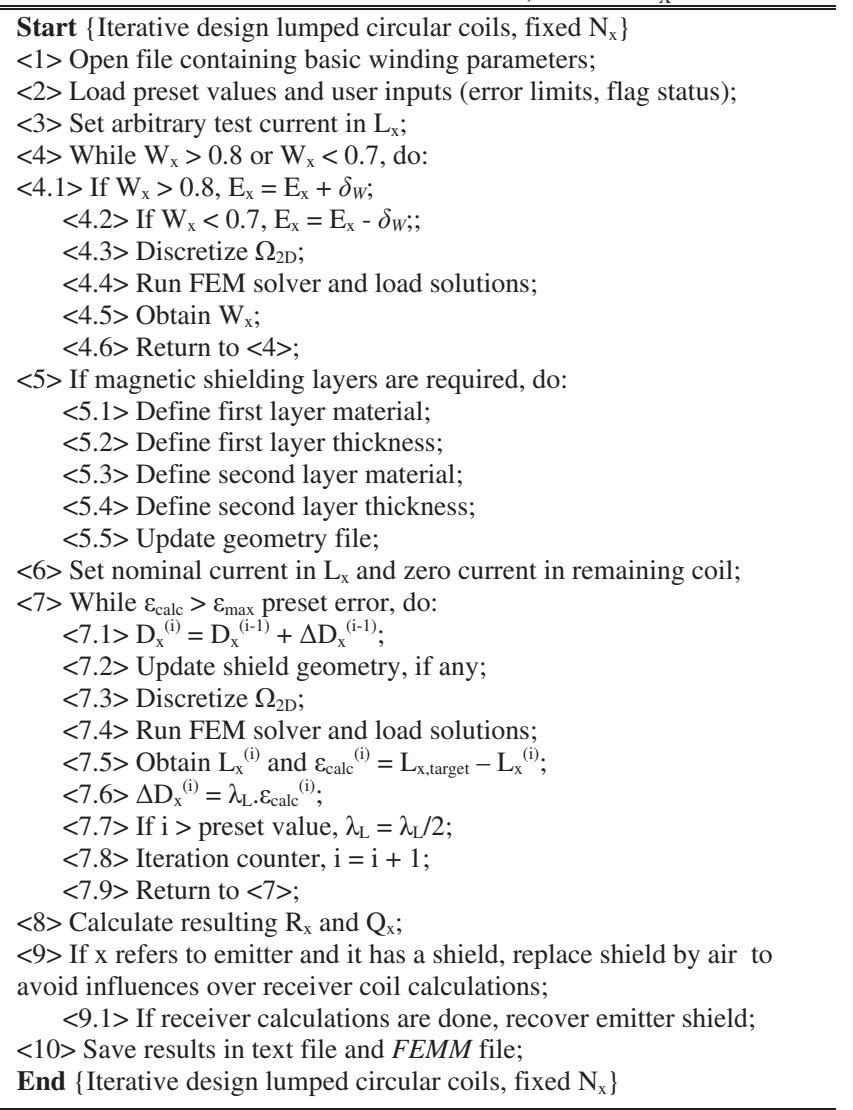

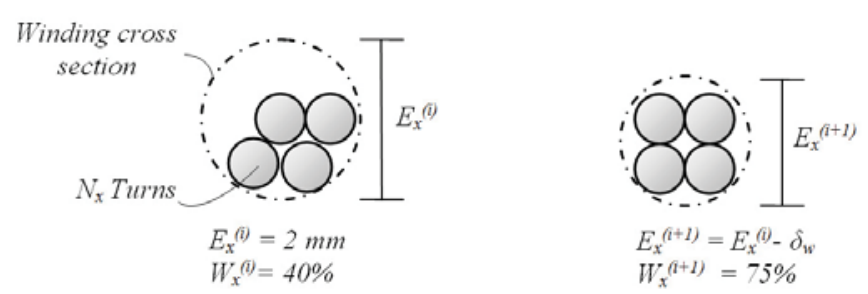

(a)

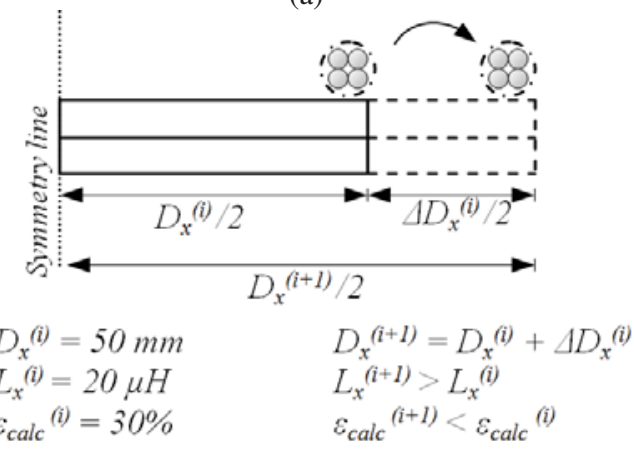

(b)

Fig. 5. Details for Algorithm I, (a) winding fill factor, $W_{x}$, iterative adjust procedure and (b) main loop with arbitrary values shown.

2) Lumped circular coils, fixed diameter - This represents a coil system with mechanical restriction being $D_{x}$ the target diameter. The procedure is depicted in Algorithm II. In this case, $N_{x}$ is the adjustable variable and it is incremented or decremented in step $<5.1>$ or $<5.2>$ depending on the sign of $\varepsilon_{\text {calc }}{ }^{(i)}$. Each time $N_{x}$ is modified, the cross section of the coil is checked to ensure $0.7 \leq W_{x} \leq 0.8$.

\section{ALGORITHM II}

LUMPED CIRCULAR COILS, FIXED $\mathrm{D}_{\mathrm{X}}$

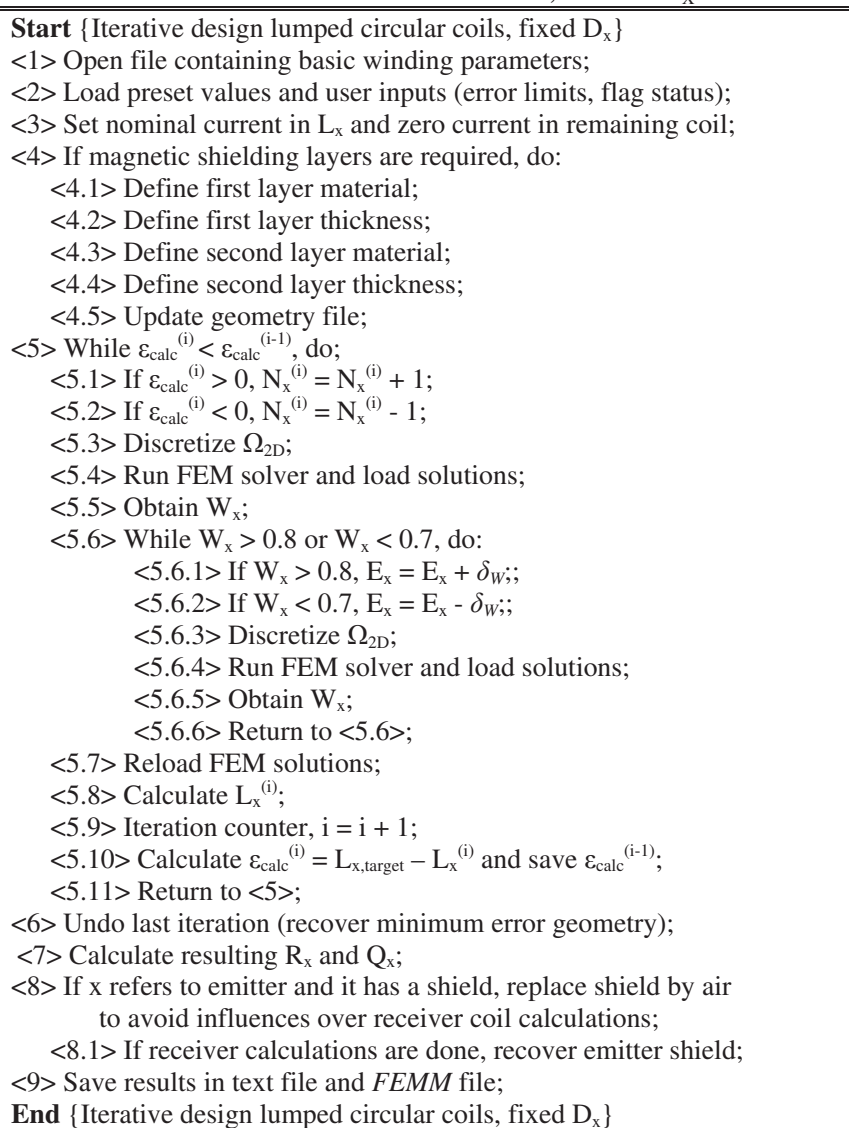




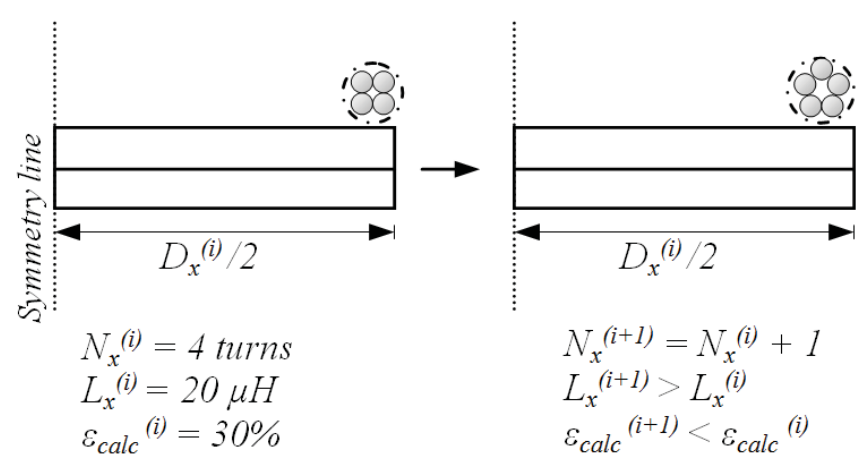

Fig. 6. Details for Algorithm II, main loop with arbitrary values shown.

The main loop from $<5.1>$ to $<5.11>$ includes the $W_{x}$ loop. Since $N_{x}$ can only assume integer values (no fractional turn allowed), it may occur that an increment results in $\varepsilon_{\text {calc }}{ }^{(i)}>$ $\varepsilon_{\text {calc }}{ }^{(i-1)}$. Thus, the main loop is finalized when the error increases between successive iterations and the geometric configuration of the iteration with lowest calculated error is maintained. Figure 6 illustrate this algorithm.

3) Spiral coils, fixed number of turns - The algorithm for this kind of winding begins by defining the cross section of one single turn based on the wire properties obtained in an earlier winding design routine.

\section{ALGORITHM III}

\section{SPIRAL COILS, FIXED $\mathrm{N}_{\mathrm{X}}$}

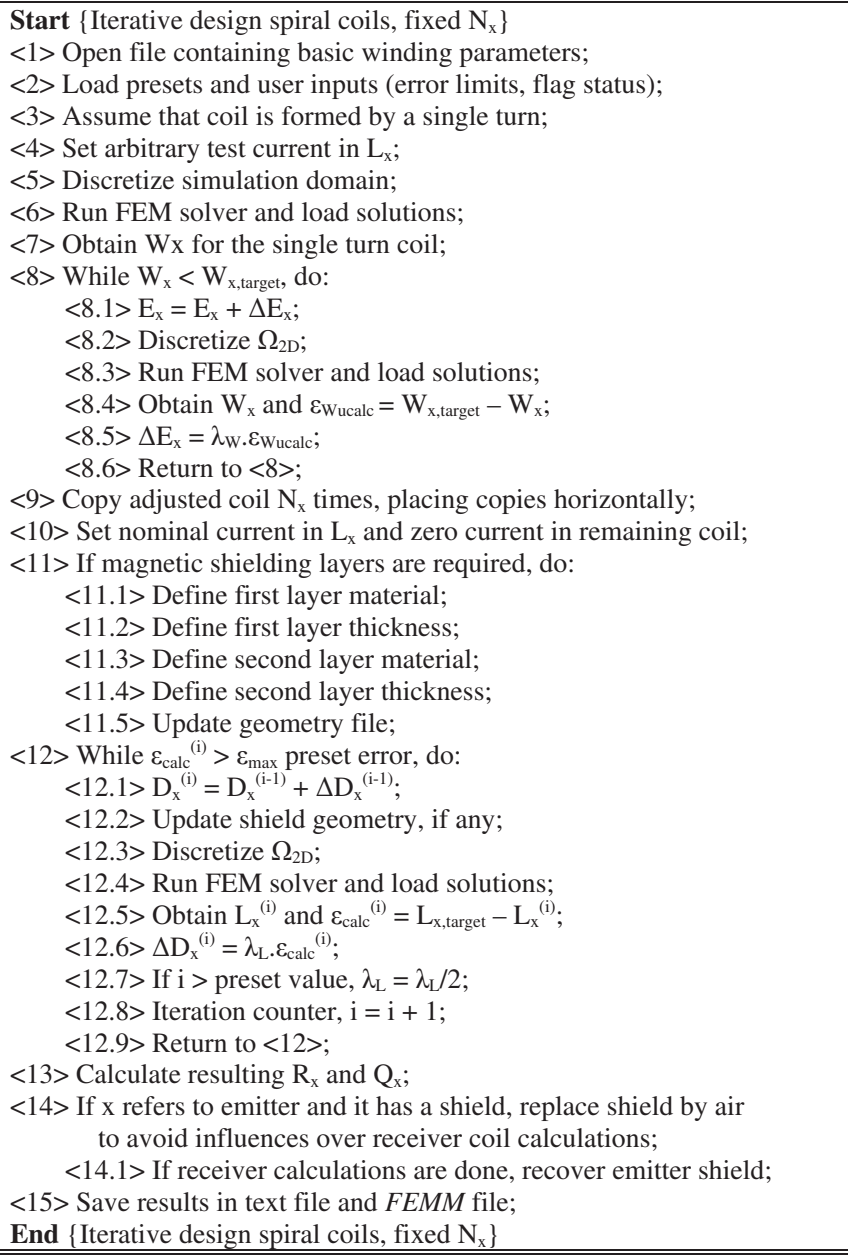

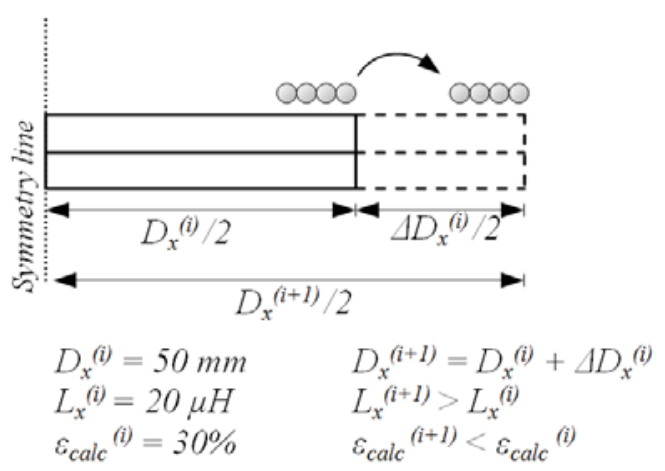

Fig. 7. Details for Algorithm III, main loop with arbitrary values.

$W_{x}$ is adjusted once (based on a target $W_{x, \text { target }}$ ) and the resulting cross section is replicated side-by-side $N_{x}$ times. The winding fill factor loop in this case was implemented with a strategy similar to that seen in Algorithm I, main loop, that is to vary $E_{x}$ according to $\Delta E_{x}$ (which is calculated with $W_{x}$ error $\varepsilon_{\text {Wucalc }}$ and a scalar winding error gain $\lambda_{W}$ ). This was done to indicate to possible users that they can add and modify sub-routines. The main loop is illustrated in Figure 7.

4) Spiral coils, fixed diameter - This case can be used to include a different mechanical restriction approach, where $D_{x}$ is understood as maximum diameter, $D_{x, \max }$, and not as target diameter (as was the case in Algorithm II).

\section{ALGORITHM IV}

\section{SPIRAL COILS, FIXED D}

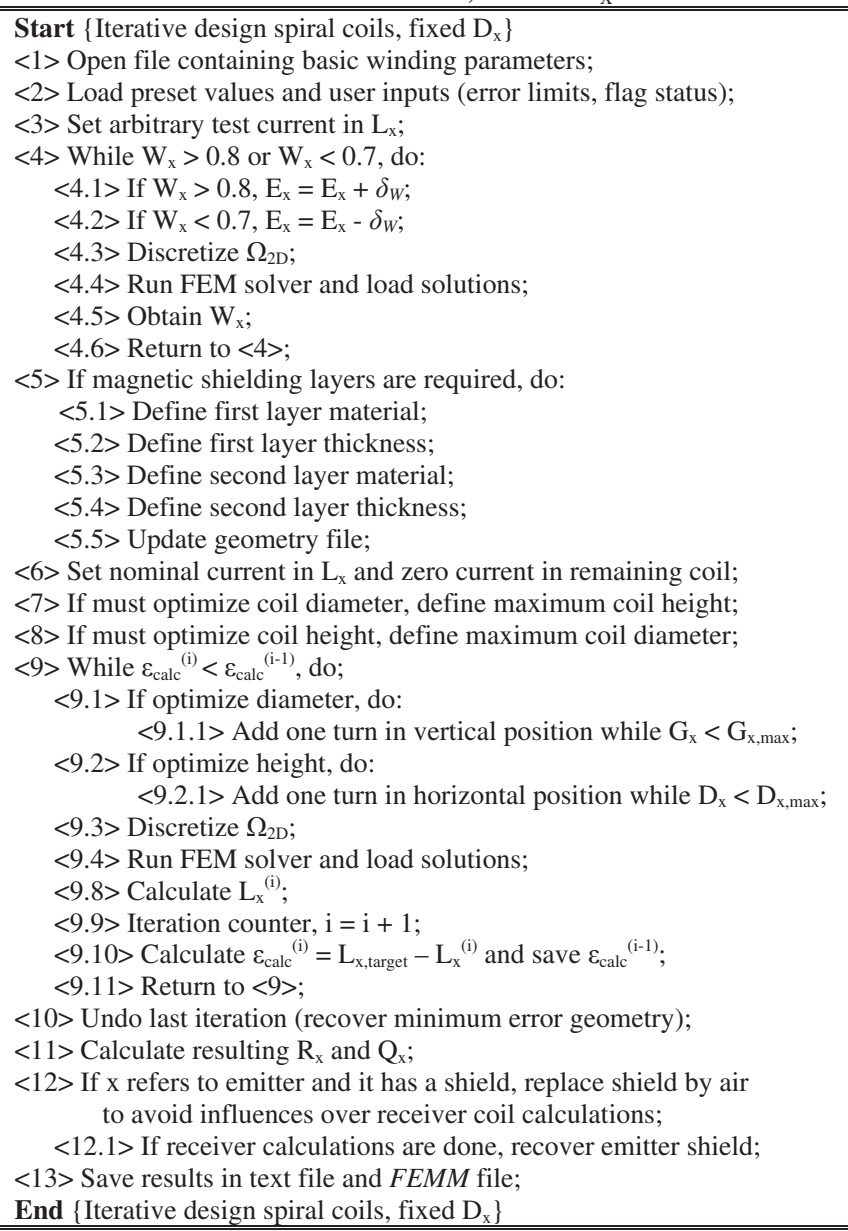


After $W_{x}$ is adjusted in steps $<4.1>$ to $<4.6>$, the user has the option to add turns in horizontal direction or in the vertical direction.

In the first possibility, $D_{x}$ is increased significantly while the coil high is kept fixed. If maximum diameter is reached and $L_{x, \text { target }}$ was not found, one vertical layer is added.In the second possibility, total coil height $G_{x}=N_{x} E_{x}$ is increased while $G_{x}<G_{x, \max }$. If $L_{x, \text { target }}$ was not found, one horizontal layer is added. So, it is possible to control the algorithm design method in order to achieve various coil shapes. Main loop alternatives are represented in Figures 8(a) and 8(b).

Finally, after using one of the discussed algorithms, since self-inductances and the coupling coefficient are known or desired, the mutual inductance can be calculated as $M=$ $k\left(L_{p} L_{s}\right)^{1 / 2}$. This analytical result of $M$ is used in a second MatLab routine that adjusts the separation distance between coils until the mutual inductance is close enough to the analytical result. In fact, using FEM the mutual inductance can be obtained by $M=V_{s a} /\left(2 \pi f_{r} \hat{I}_{p}\right)$ in terms of the peak induced open circuit voltage (obtained in FEM by solving (6)), primary current and operating frequency (which are inputs). This routine is presented in Algorithm V.

The separation distance is increased or decreased until the difference of the obtained open circuit voltage $V_{s a}$ and the reference value from the electric circuit simulation, $V_{\text {sa,target }}$, is less than a tolerance $\varepsilon_{M}$. Self-inductances are re-calculated in each distance variation. This has no effect over circular air-cored inductors, but is mandatory for coils provided of high-permeability cores or even air-cored spiral coils since the flux paths are distorted by the presence of nearby structures and $L_{x}$ becomes a function of emitter-receiver distance, $L_{x}(e)$.
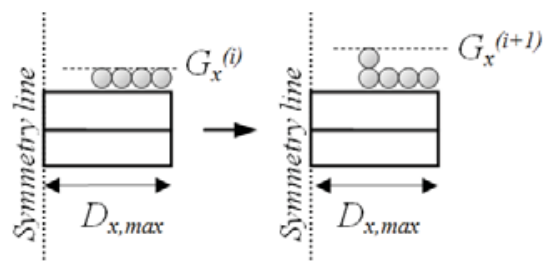

$$
\begin{aligned}
& N_{x}^{(i)}=4 \text { turns } \\
& G_{x}^{(i)}=2 \mathrm{~mm} \\
& L_{x}^{(i)}=20 \mu \mathrm{H} \\
& \varepsilon_{\text {calc }}{ }^{(i)}=30 \%
\end{aligned}
$$

$$
\begin{aligned}
& N_{x}{ }^{(i+1)}=N_{x}{ }^{(i)}+1 \\
& G_{x}^{(i+1)}=2 \mathrm{~mm}+1 \text { winding layer } \\
& L_{x}{ }^{(i+1)}>L_{x}{ }^{(i)} \\
& \varepsilon_{\text {calc }}{ }^{(i+1)}<\varepsilon_{\text {calc }}{ }^{(i)}
\end{aligned}
$$

(a)
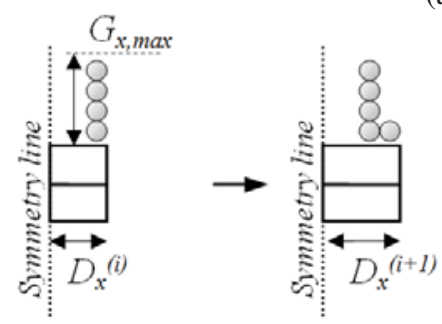

$N_{x}^{(i)}=4$ turns

$D_{x}^{(i)}=10 \mathrm{~mm}$

$L_{x}^{(i)}=20 \mu \mathrm{H}$

$\varepsilon_{\text {calc }}{ }^{(i)}=30 \%$

$$
\begin{aligned}
& N_{x}{ }^{(i+1)}=N_{x}^{(i)}+1 \\
& D_{x}{ }^{(i+1)}=10 \mathrm{~mm}+1 \text { winding layer } \\
& L_{x}{ }^{(i+1)}>L_{x}{ }^{(i)} \\
& \varepsilon_{\text {calc }}{ }^{(i+1)}<\varepsilon_{\text {calc }}{ }^{(i)}
\end{aligned}
$$

Fig. 8. Details for Algorithm IV, (a) total coil height optimization and (b) coil diameter optimization.

\section{ALGORITHM V}

\section{MUTUAL INDUCTANCE REPRESENTATION}

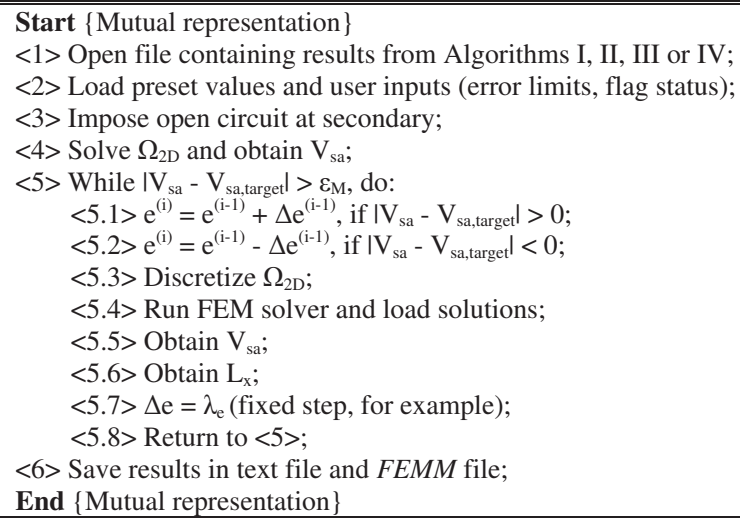

By the end of the iterative procedure, the large number of initial electrical parameters has been accurately put into a magnetic representation which allows the analysis of the loosely coupled system. Considering that non-linear magnetic materials, high-complexity geometries, skin effect, proximity effect and realistic structures can be fully simulated with the iterative procedures, it can be said that their uses are far beyond the possibilities of analytical, semianalytical or empirical approaches for IPT design. Thus, the method descriptions made so far in the present paper are novel contributions themselves.

\section{CHARACTERIZATION OF AXIAL, LATERAL AND ANGULAR MISALIGNMENT}

In practical applications of IPT converters there are natural misalignments that result from the usual operation of the converter. Different applications are subjected to different levels of coil misalignment:

In electric vehicles, as the receiver coil is fixed at the bottom of the vehicle and the elevation of the vehicle in respect to the ground (where the emitter coil is located) is also fixed by the wheels, the loosely coupled system has some natural immunity to axial misalignment (constant separation distance). However, IPT converters are subjected to high lateral misalignments due to the difficulty to park the vehicle perfectly centered with the emitter coil [9];

In biomedical implants, such as cochlear devices and pacemakers, converters must be adjusted to the user characteristics. This is because the separation distance varies from user to user due the length of biological tissues and bones. These applications are also subjected to high lateral misalignments. In ocular implants, the converter requires large angular tolerance due to the eye movement, that significantly contributes to the reduction of coupling coefficient [10];

In portable consumer electronic devices, such as cell phones, tablets and laptops, if a high degree of mobility is desired the converter is usually under the influence of axial, lateral and angular misalignments [11].

From the above comments, one can clearly conclude that the operation of IPT converters under any kind of misalignment leads to reduction of coupling coefficient and a consequent reduction in power transfers according to (2). Thus, the Finite Element Method is again applied to evaluate 
$k$ as a function of $d_{c}, e$ and $\alpha$, namely the lateral, axial and angular misalignment variables, respectively. Since a $k\left(e, d_{c}, \alpha\right)$ function can be obtained, the loosely coupled system can be completely characterized in terms of power and transfer efficiency.

For the experimental results shown in next topics, the setup presented in Figure 9 was used. In the setup, three 1 $\mathrm{mH}$ (target) air-core inductors were implemented, each one with a different diameter (thus, resulting in different number of turns): For inductor 1, $L_{x}=1.007 \mathrm{mH}, D_{x}=50 \mathrm{~mm}$; For inductor 2 and 4, $L_{x}=1.039 \mathrm{mH}$ and $D_{x}=100 \mathrm{~mm}$; For inductor $3, L_{x}=1.028 \mathrm{mH}$ and $D_{x}=200 \mathrm{~mm}$. In all situations, $f_{r}=20 \mathrm{kHz}, \hat{I}_{p}=10 \mathrm{~A}$.

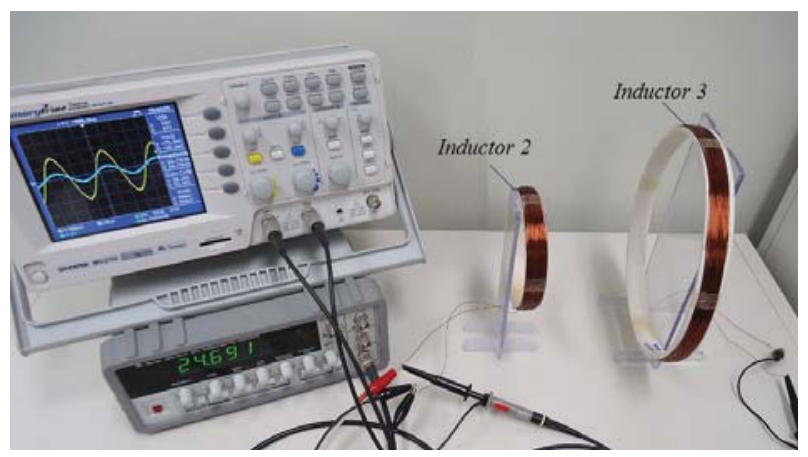

Fig. 9. Experimental setup.

\section{A. Lateral Misalignment Analysis}

In this analysis, $d_{c}$ (refer to Figure 2) is varied while $e$ is kept fixed at a chosen value. Coils are parallel to each other, $\alpha=0^{\circ}$. It must be mentioned that, since the simulation procedure is not axisymmetric, the $2 \mathrm{D}$ planar representation must be obtained. This is automatically done by the proposed MatLab algorithms.

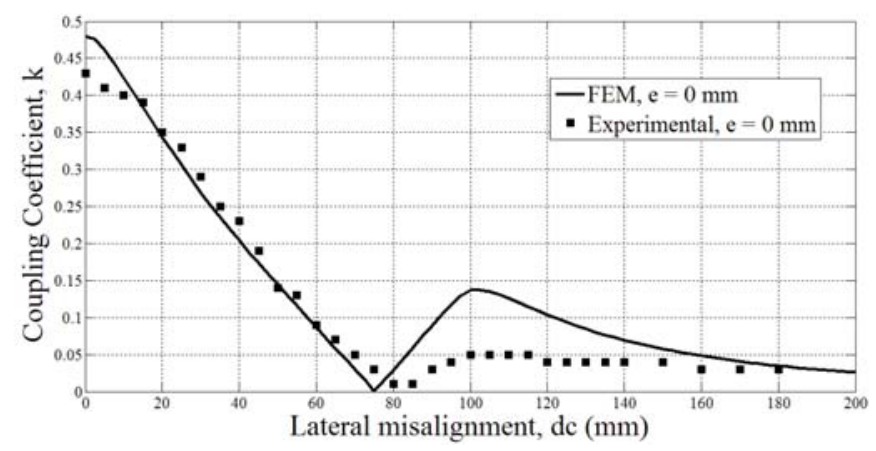

Fig. 10. Lateral misalignment simulation and experimental results.

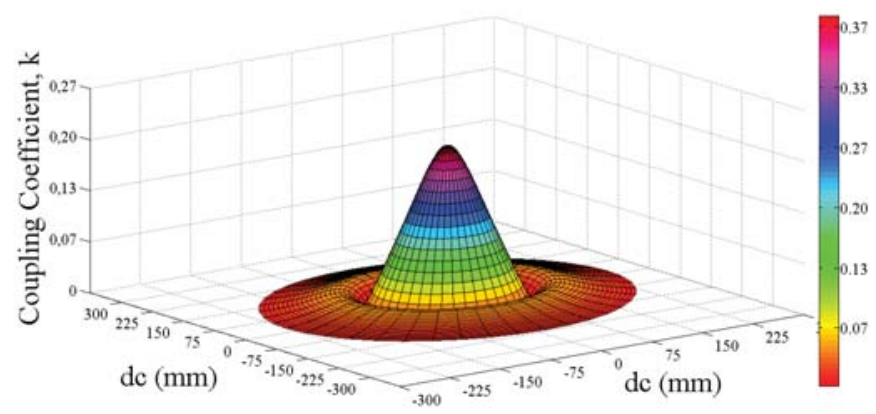

Fig. 11. A 3D user-friendly $k$ mapping example.
Figure 10 shows the results for the hypothetical loosely coupled system and compares simulated and experimental data obtained with inductors 2 and 4 . One can observe that $k$ is maximum when $d_{c}=0$ and it decreases significantly if the receiver coil is moved laterally from the emitter. The error observed between experimental and FEM results are explained by the experimental setup positioning mechanism. Anyway, the experimental data is enough to prove the concept and are in agreement with literature data [9].

A user-friendly tridimensional map of the coupling coefficient can be also obtained, as shown in Figure 11. Different geometries present different tolerances to lateral misalignment. Planar transformers can also be simulated with the proposed strategy and they are a good alternative to achieve better spatial magnetic flux distribution.

\section{B. Axial Misalignment Analysis}

For axial misalignment, the coupling coefficient is calculated while separation distance $e$ is increased, $d_{c}=0$ (coils are kept concentric) and $\alpha=0^{\circ}$ (coils are kept parallel to each other). The increment of $e$ can be adjusted as desired and results shown in Figure 12 were obtained with increments of $1 \mathrm{~mm}$. For each separation distance a new simulation is done and results are saved by the proposed algorithm. Using the FEM 2D formulation, these simulations are completed in less than one minute in a conventional personal computer.

In Figure 12, using $D_{p}=100 \mathrm{~mm}$, combinations $D_{p}=D_{s}$, $D_{p}=2 D_{s}$ and $D_{p}=D_{s} / 2$ were subjected to axial coil misalignment. In all cases, $L_{p}=L_{s}$. The only different among inductors are the diameters and as a result, the number of turns. One can observe that experimental results fit very well their respective simulated curve. However, the most important is that a non-trivial result is obtained: a characterization of coupling coefficient decay behavior for different coil geometries. It is very intuitive that $k$ is maximum for $e=0$. When $D_{p}=D_{s}, k$ reach its highest value, of 0.48 , but it is reduced significantly at each increment in separation distance. Instead, for $D_{p}=D_{s} / 2$ (condition shown in Figure 9), the initial $k$ is only 0.21 , but its reduction is linear and less sensitive to $e$ variations. The result is even better as the ratio $D_{p} / D_{s}$ decreases.

By perfectly understanding the behavior of a loosely coupled system, the converter's control strategy can be better designed to compensate the coupling coefficient variation curve, thus helping to optimize power transfer.

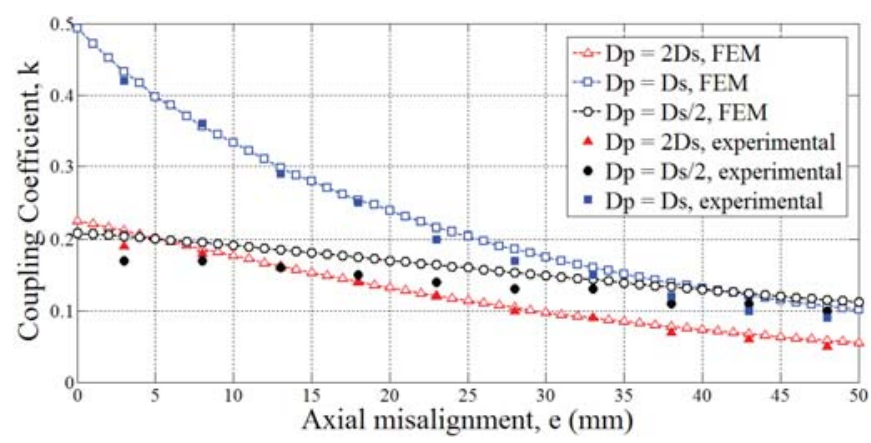

Fig. 12. Axial misalignment simulation and experimental results comparison. 


\section{Angular Misalignment Analysis}

Angular misalignment can also be characterized for a large number of coil configurations. Separation distance is fixed at the desired distance while $\alpha$ is varied from $0^{\circ}$ to $180^{\circ}$. This can be done for any $d_{c}$ condition. Also the increment of $\alpha$ can be adjusted as desired. In Figure 13, results were obtained for an increment of $5^{\circ}$ between simulations. It can be seen that $k$ is very dependent on $\alpha$ for the hypothetical geometry. Also, depending on the condition of lateral misalignment $\left(d_{c} \neq 0\right), k$ can be reduced to zero (no power transfer from emitter to receiver).

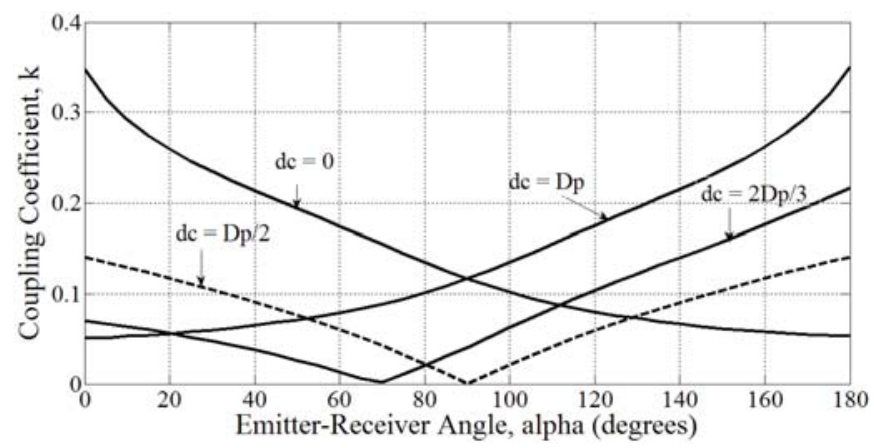

Fig. 13. Angular misalignment simulation result.

The three kinds of misalignment described can be studied for a given loosely coupled system, at the same time, in order to achieve a complete spatial characterization. When $k$ is predicted under various spatial conditions, the power transfer can be simply determined by solving (2).

The algorithms proposed in this paper also allow the operating frequency to be varied. This is especially useful to predict coils quality factor or determine rather a magnetic core has acceptable frequency response or not under transient conditions. There is no record in literature that these approaches can be carried out with analytical formulation, even for in-depth formulations [12].

\section{OPTIMIZATION OF LOOSELY COUPLED SYSTEMS}

Since $L_{x}\left(N_{x}, D_{x}\right)$ has an infinite number of possible solutions if no restrictions are imposed, analytical, semianalytical and empirical approaches cannot easily indicate the optimized $N_{x}$ or $D_{x}$ for a given target $L_{x}$ that maximizes $k\left(e, d_{c}, \alpha\right)$ function. In fact, only a computational method is able to perform such a complex combinatory analysis in practical time.

To provide a solution for this problem, an optimization routine was implemented and its objective is to indicate the best coil diameter for emitter and receiver, in a way that $k$ is maximized for a given separation distance of interest. Basically, the algorithm shown in Figure 5 is executed $n$ times for different input $N_{x}$ values, but target self-inductances $L_{p}$ and $L_{s}$ are kept the same (notice that it is possible to have $L_{p}=$ $L_{s}$ or $L_{p} \neq L_{s}$ ). The result is a matrix containing $n$ possible geometrical representations for $L_{p}$ and $L_{s}$.

Thus, a combinatory analysis is made as follows: First MatLab creates geometrical entities in blank FEMM files and represent every possible combination of $D_{p}$ and $D_{s}$ (one FEMM file for each combination), making sure that emitter and receiver are separated by the user-defined distance. This will result in $n^{2}$ files. Then, each combination is discretized, simulated and post-processed. Coupling coefficient is calculated for each possible solution. The raw data is organized in a user-friendly graph, that indicates optimized $D_{p}$ and $D_{s}$ that maximize $k$ (or $M$ ).
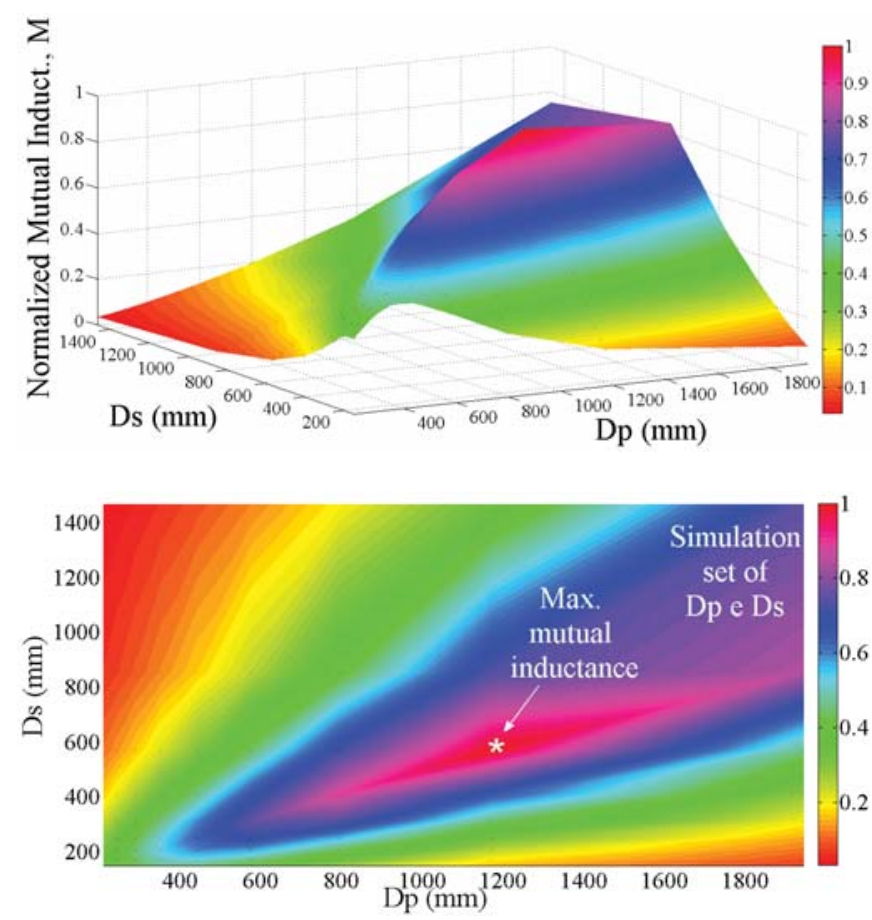

Fig. 14. Examples of optimization map obtained for $L_{p} \neq L_{s}$. Color map represents normalized mutual inductance.

Figure 14 shows the behavior of mutual inductance (normalized for simplicity) for some possible combinations of $D_{s}$ and $D_{p}$ for a hypothetical loosely coupled system where $L_{p}=3 L_{s} / 2$ at an arbitrary (but fixed) separation distance. Again, if the best $D_{p} / D_{s}$ ratio is chosen, power transfer will be optimized.

\section{SAFETY LIMITS MAPPING}

As IPT converters usually make use of high frequency and high current converters, it is possible that significant magnetic field intensity $|H|$ result from its operation. Based on [13], which provides limits for general public exposure as a function of $f_{r}$, a dedicated routine calculates the maximum reference magnetic field allowed $H_{\text {ref }}$ and indicates those spatial coordinates that are in agreement with the safety guideline. This is done by reading FEMM simulation results point-by-point in the simulation domain and saving in a MatLab matrix for further processing. The resolution of the obtained map depends on FEM mesh size, which is determined automatically according to the materials present in the simulation domain and its dimensions. An example is shown in Figure 15, where an axisymmetric converter was simulated and coordinates where $|H|>H_{\text {ref }}$ were hatched. Here, $H_{\text {ref }}$ is $27 \mu \mathrm{T}$, according to [13] for $f_{r}=20 \mathrm{kHz}$. This helps the user to spatially preview and analyze the area under influence of the converter and then add or improve magnetic shields by running the routine once more. 


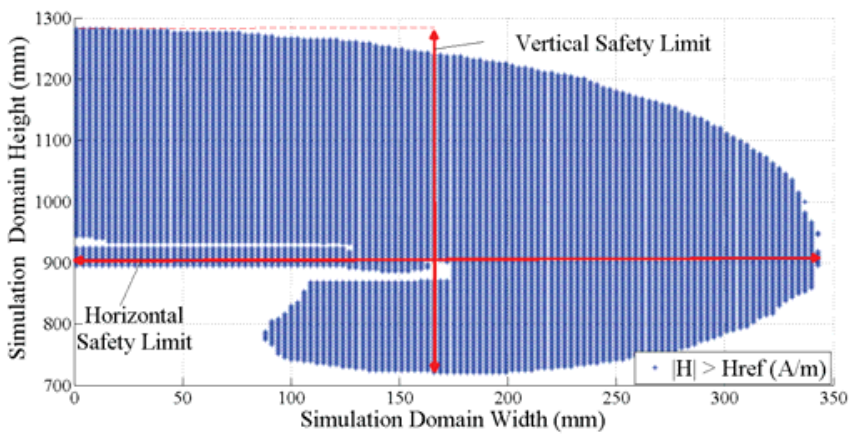

Fig. 15. ICNIRP general public exposure limits (magnetic field intensity $\mid \mathrm{HI}$ ) mapped around a hypothetical converter (half simulation domain is shown).

In Figure 15, the converter (at its maximum excitation parameters) is said to be in accordance with [13] if no general public is present within the area defined by the indicated horizontal and vertical limits.

\section{CONCLUSIONS}

This paper described several computational tools based on finite element method intended to help and guide the design of loosely coupled inductors system for inductive power transfer converters. Compared to currently available analytical approaches, the iterative method is much more versatile from the point of view that the proposed algorithms can evaluate any condition of $d_{c}, e, \alpha$ or even combinations of these three parameters at the same time for fully editable circular and spiral coil systems. Iterative routines demand minimum computational resources since they are based on 2D FEM formulation, thus resulting in a more attractive method. Also, graphical visualizations of the phenomena occurring in loosely coupled system (such as flux paths, saturation, flux density, magnetic field intensity) are provided, then allowing the designer to further investigate and improve the concept without previous or in-depth experience with FEM. Moreover, the proposed tools provide an opportunity to investigate optimization of coil geometries and safety limits around the converter, based on international standard. The above mentioned are considered unique contributions of the present work and advantages over existing design methods. Finally, this powerful tool allows modifications to include other routines and post-processor functions dedicated to specific IPT applications.

\section{REFERENCES}

[1] M. Hutin, M. LeBlanc, "Transformer system for electric railway", US 527.857, Oct. 1894.

[2] Y. S. Seo, Z. Hughes, M. Hoang, D. Isom, "Investigation of Wireless Power Transfer in Throughwall Applications", in Proc. of Asia-Pacific Microwave Conference, pp. 403-405, 2012.

[3] M. K. Kazimierczuk, D. Czarkowski, Resonant power converters, John Wiley \& Sons, $2^{\text {nd }}$ ed., New Jersey, USA, 2010.

[4] A. P. Hu, Wireless/Contactless power supply, VDM, $1^{\text {st }}$ ed., Saarbrücken, 2009.
[5] A. C. M. de Queiroz, "Cálculo de indutâncias e indutâncias mútuas pelo método de Maxwell", Semana da Eletrônica 2003, Universidade Federal do Rio de Janeiro, Rio de Janeiro, pp. 1-7, 2003.

[6] F. W. Grover, Inductance Calculations, Dover Publications, $1^{\text {st }}$ Edition, New York, USA, 2009.

[7] D. Meeker (2012). FEMM - Finite element method magnetics (Version 4.2) "Software". Available at www.femm.info. Accessed 01/02/2014.

[8] J. W. Arthur, "An elementary view of Maxwell's displacement current", IEEE Antennas and Propagation Magazine, vol. 51, no. 6, pp 58-68, 2009.

[9] G. A Covic, J. T. Boys, M. L. G. Kissin, H. G. Lu, “A three-phase inductive power transfer system for roadway-powered vehicles", IEEE Transactions on Industrial Electronics, vol. 54, no. 6, pp. 3370-3378, Dec., 2007.

[10] Y. Zhao, M. Nandra, C. Yu, Y. Tai, "High performance 3-coil wireless power transfer system for the 512electrode epiretinal prosthesis", in Proc. IEEE EMBS, pp. 6583-6586, 2012.

[11]E. Waffenschmidt, T. Staring, "Limitation of Inductive Power Transfer for Consumer Applications", in Proc. of IEEE European Conference on Power Electronics and Application, pp. 1-10, 2009.

[12] K. Fotopoulou, B. W. Flynn, "Wireless Power transfer in loosely coupled links: coil misalignment model", IEEE Transactions on Magnetics, vol. 47, no. 2, pp. 416-430, Feb., 2011.

[13] International Commission on Non-Ionizing Radiation Protection, ICNIRP Guidelines for Limiting Exposure to Time-Varying Electric and Magnetic Fields (1 Hz - 100 kHz, 2010.

\section{BIOGRAPHIES}

Azauri Albano de Oliveira Jr. was born in 1955 . He graduated in electrical/electronic engineering in 1977 from Escola de Engenharia de São Carlos (EESC), Universidade de São Paulo (USP), where he also obtained the MSc. degree in electrical engineering in 1984. He concluded his DSc. in electrical engineering in 1991 in Escola Politécnica, Universidade de São Paulo.

Since 1978, he is professor at the Electrical and Computing Engineering Department, EESC, USP where he is the current coordinator of Power Electronics and Control Laboratory. His research interests include power electronics, electric machinery drives and engineering education.

Rodolfo Castanho Fernandes was born in 1986. He graduated in electrical engineering from Univ. Estadual Paulista (UNESP), Ilha Solteira, SP, in 2008. He obtained the MSc. degree in power electronics in 2010 from the same institution. Currently, he is working towards the DSc. degree in dynamical systems at Escola de Engenharia de São Carlos, Universidade de São Paulo.

Since 2010 he is with Brazilian heavy automotive industry where he is involved in research and development programs on heavy machinery automation, portable battery operated products and power converters. 